\title{
Angstrom-Scale Distance Dependence of Antenna-Enhanced Vibrational Signals
}

\author{
Jörg Bochterle, ${ }^{\dagger}$ Frank Neubrech, ${ }^{\dagger}$ Tadaaki Nagao, ${ }^{\dagger}$ and Annemarie Pucci ${ }^{\dagger, *}$ \\ ${ }^{\dagger}$ Kirchhoff Institute for Physics, University of Heidelberg, Im Neuenheimer Feld 227, 69120 Heidelberg, Germany and ${ }^{\ddagger}$ World Premier International (WPI) Research \\ Center for Materials Nanoarchitectonics (MANA), National Institute for Materials Science (NIMS), 1-1 Namiki, Tsukuba, Ibaraki, 305-0044, Japan
}

\begin{abstract}
The resonantly enhanced near-field of micrometer-sized gold antennas has been probed with Angstrom-scale resolution. In situ surfaceenhanced infrared spectroscopic vibrational signals of carbon monoxide (CO) layers cold-condensed on the antennas in ultrahigh-vacuum conditions are compared to the signals of $\mathrm{CO}$ layers with corresponding thicknesses on a flat gold surface. Vibrational signals of $\mathrm{CO}$ as well as the shift of the plasmonic resonance frequency were used to analyze the distance dependence of the near-field. The signal enhancement induced by the antennas not only decays
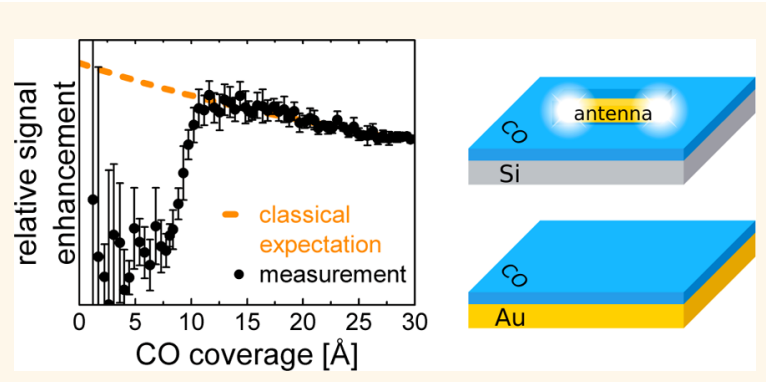
monotonically from the surface but, in contrast to classical near-field models, shows a maximum between 10 and $15 \AA$ and decays also toward the surface of the antenna. This effect is attributed to the spill-out of the electron wave function, as expected from quantum mechanical calculations.
\end{abstract}

KEYWORDS: local near-field · quantum plasmonics $\cdot$ gold antennas $\cdot$ cold-condensed carbon monoxide $\cdot$ SEIRS

S urface-enhanced spectroscopic methods, such as surface-enhanced Raman scattering (SERS), ${ }^{1-4}$ surface-enhanced infrared spectroscopy (SEIRS), ${ }^{5-7}$ and surfaceenhanced fluorescence, ${ }^{8,9}$ have gained large interest in the field of life science due to their high sensitivity. They are of great importance for example in biology, ${ }^{10}$ medicine, ${ }^{11}$ and food and water safety, ${ }^{12}$ as well as for toxics ${ }^{13}$ and explosives detection, ${ }^{14}$ where the detection of minute amounts of molecular species is essential. The enhancing mechanism is provided by the interaction of molecular excitations with plasmonic excitations of metallic nanostructures. These localized surface plasmon resonances (LSPRs) cause considerable near-field enhancement, which allows extraordinarily high sensitivity. For optimized enhancement in sensing applications, as well as for fundamental physical questions, ${ }^{15-18}$ the spatial near-field distribution around the surface of a nanoparticle is of great importance. A commonly used method to measure local fields is scanning near-field optical microscopy (SNOM), where light is scattered out of the near-field by means of a dielectric tip. ${ }^{19}$ This technique has the advantage of providing information in all three dimensions, ${ }^{20}$ but resolution is limited by the radius of the tip apex (a few nanometers) and thus not high enough to measure the near-field distance dependence on the Angstrom scale. This regime is of especially high interest since classic electrodynamical and quantum-dynamical models predict striking differences in the near-field.

Classical Predictions. The local field distribution is usually simulated by solving Maxwell's equations with the corresponding boundary conditions. Such simulations use dielectric functions to describe the response of homogeneous materials with unrealistically sharp boundaries and do not consider structure on an atomic level. These simulations yield a monotonic field increase up to the highest field directly at the surface. In conclusion, the most sensitive area of the nanoparticle is expected directly at its surface. ${ }^{21}$

Quantum Mechanical Predictions. Recent theoretical studies ${ }^{17,22-25}$ beyond classical limits however found that the quantum mechanical nature of conduction electrons can yield deviations from a classical description at the subnanometer scale. A changed dispersion curve of the surface plasmon, ${ }^{24-26}$ tunneling of electrons between nanoparticle dimers $^{17,22}$ and for single particles a decreased electric field at the metal surface due to electron spill-out and screening ${ }^{23}$ have been reported. These deviations from the classically derived near-field behavior is
* Address correspondence to pucci@kip.uni-heidelberg.de.

Received for review September 19, 2012 and accepted November 19, 2012.

Published online November 19, 2012 10.1021/nn304341c

C 2012 American Chemical Society 
predicted to be relevant up to distances of about $5 \AA$ from the surface ${ }^{23}$ of the simulated, ideally smooth particles, being only a few nanometers in size. For experimentally feasible particles, with rough surfaces and realistic sizes, a closer spacing of electronic states pushes the edge of the quantum regime even further away from the surface. ${ }^{23}$

Near-field probes with atomic dimensions, such as molecules, are small enough to prove such quantum effects experimentally when positioned close to the metal surface with subnanometer precision.

Refractive Index Sensing. A commonly used method to detect changes in the surrounding of nanoparticles exploits the shift of the LSPR frequency upon changing the refractive index of the surrounding or by varying the thickness of a thin film on the surface of small metal particles. ${ }^{27}$ In the latter case, the shift gives information about the near-field distribution perpendicular to the surface. Layers with defined thickness can be realized either by self-assembled monolayers (SAM) ${ }^{28,29}$ or by atomic layer deposition (ALD). ${ }^{30}$ These studies report a linearly increasing shift of the LSPR frequency for the first $50 \AA$, corresponding to an almost constant nearfield. The thickness control for ALD is promising, but unfortunately the measurements were performed ex situ, resulting in large errors due to sample positioning.

Local Molecular Excitations. A second approach to probe the field distribution is to place molecules close to the particle's surface and use their antennaenhanced excitations as a local probe. Fluorescence, ${ }^{31}$ Raman signals (SERS), ${ }^{32}$ or infrared (IR)-active vibrational modes (SEIRS) ${ }^{5}$ of molecules could be considered possible measures, but for very small distances $(<50 \AA)$, fluorescent molecules are not suitable as local field sensors due to quenching. ${ }^{31}$

In SERS measurements of adsorbed molecules, it is well known that the enhanced near-field gives not only signal enhancement but also some less clear chemical effects, involving charge transfer between substrate and adsorbate. ${ }^{33}$ Both effects can be separated by introducing an isolating spacer layer. The measurements yield monotonically increasing signals for decreasing spacer thickness. ${ }^{32,34}$ This method is promising, but data points below $10 \AA$ spacer thickness are not available in the literature.

However, SEIRS seems to be the most promising approach to precisely measure the near-field distribution. In an early work ${ }^{35}$ rough metal films were used as a substrate with rather large SEIRS molecules, yielding a poor spatial resolution of $25 \AA$.

This Work. We present SEIRS measurements using micrometer-sized lithographic gold antennas, featuring resonances in the IR-spectral range to map the distance dependence of the near-field. In order to obtain the high resolution that is needed to resolve the deviations induced by electron spill-out, we used carbon monoxide (CO) as probe molecule. The CO multilayer growth at $18 \mathrm{~K}$ allows thickness resolution well below $10 \AA$ and changes the electronic structure of the nanoantenna surface only slightly, due to the absence of chemical bonds, allowing us to interpret the observed enhancement to be solely caused electromagnetically.

A further great advantage of our SEIRS experiments is the measurement of two quantities at the same time, giving independent information. On one hand, we look at the enhanced vibrational signal of the $\mathrm{CO}$ molecules, but on the other hand, we observe how the adsorbed CO layer changes the refractive index around the metallic antenna.

\section{RESULTS AND DISCUSSION}

In the first step, we consider the different signals that appear in our measurements on the example of a CO layer thickness of $d_{\mathrm{CO}}=29.5 \AA$. Later we will explain how to extract the information of interest from these signals and apply this procedure to all spectra at different layer thicknesses. On the basis of the distancedependent evolution of the signal, we can draw conclusions about the distribution of the near-field.

IR Spectral Response. In conventional absorption spectroscopy, without any antennas, IR light is absorbed by molecular vibrations with changing dipole moments and results in a spectrally narrow dip at the vibrational frequency. In contrast, micrometer-sized metallic antennas mainly scatter IR radiation, resulting in a broad band decrease of transmitted light. For molecules adsorbed on antennas, both effects can couple, which results in a Fano-type spectral feature. ${ }^{5,36}$ In our experiment we observe all three cases, as presented in Figure 1.

The black spectrum $\left(A_{\|} / S_{\|}\right)$shows the broad LSPR of the cooled antennas with parallel (II) polarization, whereas no LSPR is excited with perpendicular $(\perp)$ polarization (black spectrum $A_{\perp} / S_{\perp}$ ). When cold-condensing $\mathrm{CO}$ on the sample at $18 \mathrm{~K}$, from a supersaturated partial pressure in the ultrahigh-vacuum (UHV) chamber, it accumulates on all surfaces with the same thickness $\left(d_{\mathrm{cO}}\right)$, i.e., on the antennas as well as on the silicon substrate. For $\perp$ polarization the signal originating from the not resonantly excited antennas is negligible since the relative area covered by gold antennas within the array is only about $1 \%$. Therefore, we see at $2139 \mathrm{~cm}^{-1}$ only the vibrational signal of CO physisorbed on the silicon substrate (blue spectrum $\mathrm{C}_{\perp} / \mathrm{S}_{\perp}$ ). For II polarization the LSPR couples to the CO vibration, resulting in a Fano-type line shape at the vibrational frequency (blue spectrum $\mathrm{C}_{\|} / \mathrm{S}_{\|}$). Due to the change of the polarizability of the antennas' surrounding, the LSPR frequency shifts toward lower energies (redshift, $\left.\Delta \omega_{\text {res }}\right)$.

For a detailed discussion on the distance dependence of the field enhancement, we now consider the 

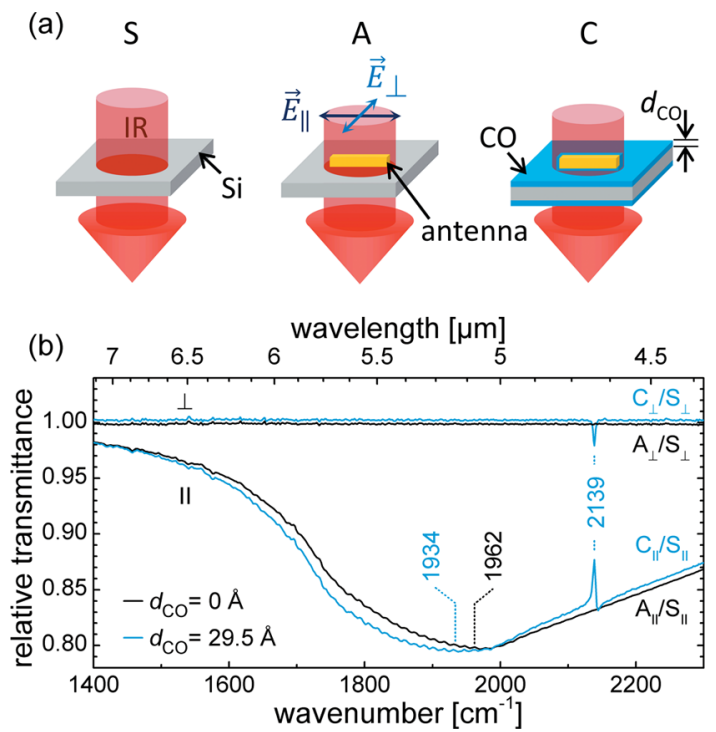

Figure 1. Schematic illustration of the measurement geometries, one antenna representing an array of antennas. S, $A$, and $C$ correspond to bare silicon substrate, gold antennas on silicon substrate, and the whole sample covered with a cold-condensed $\mathrm{CO}$ layer. The polarization of the incoming IR light is labeled parallel (II) and perpendicular $(\perp)$ to the antennas' long axis (a). Representative relative transmittance spectrum of the gold antennas on silicon substrate without (black) and with (blue) a CO layer $\left(d_{\mathrm{CO}}=29.5 \AA\right.$ ) covering the whole sample (b). For all spectra the reference has been taken on the bare silicon substrate. The resonance position of the plasmon, as obtained from second-order polynomial fits, and the position of the $\mathrm{CO}$ vibrational signal are marked.

shift of the LSPR frequency and the signal size of the CO stretch vibration separately. Since the relative transmittance changes in Figure 1 are rather small and the total plasmon shift can be only imprecisely determined, we normalized $C_{\|}$to $A_{\|}$, to better visualize the changes solely induced by the CO layer. The resulting spectrum is shown in Figure 2 a.

LSPR Shift. The red-shift of the LSPR frequency results in a broad decrease (Figure $2 \mathrm{a}, J_{\text {shift }}$ ) of the signal left of the resonance frequency and a broad increase (Figure 2a, $J^{\prime}$ shift) on the high-energy side, which is distorted by the narrow band Fano-type CO vibration. It is easily shown that the area $J_{\text {shift }}$ or $J^{\prime}$ shift is proportional to the peak shift $\Delta \omega_{\text {res, }}$ as long as $\Delta \omega_{\text {res }}$ is small compared to the spectral width of the LSPR. We evaluated the area $J_{\text {shift }}\left(d_{\mathrm{CO}}\right)$ for all different layer thicknesses and calculated the corresponding shift $\Delta \omega_{\text {res }}\left(d_{\mathrm{CO}}\right)$ by relating the total area $J_{\text {shift }}(29.5 \AA$ ) (Figure $2 \mathrm{a}$ ) to the total shift $\Delta \omega_{\text {res }}(29.5 \AA)=28 \mathrm{~cm}^{-1}$ (from 1962 to $1934 \mathrm{~cm}^{-1}$, see Figure 1). The results are plotted in Figure $4 a$ and will be discussed later.

co Vibration. To focus on the asymmetric line shape of the $\mathrm{CO}$ vibrational signal, Figure $2 \mathrm{~b}$ shows a zoom-in of Figure $2 \mathrm{a}$. The $\mathrm{CO}$ vibrational signal in Figure $2 \mathrm{~b}$ is a superposition of two $\mathrm{CO}$ vibrational signals originating from different sites on the sample. One fraction originates from the coupling with the antennas and has the

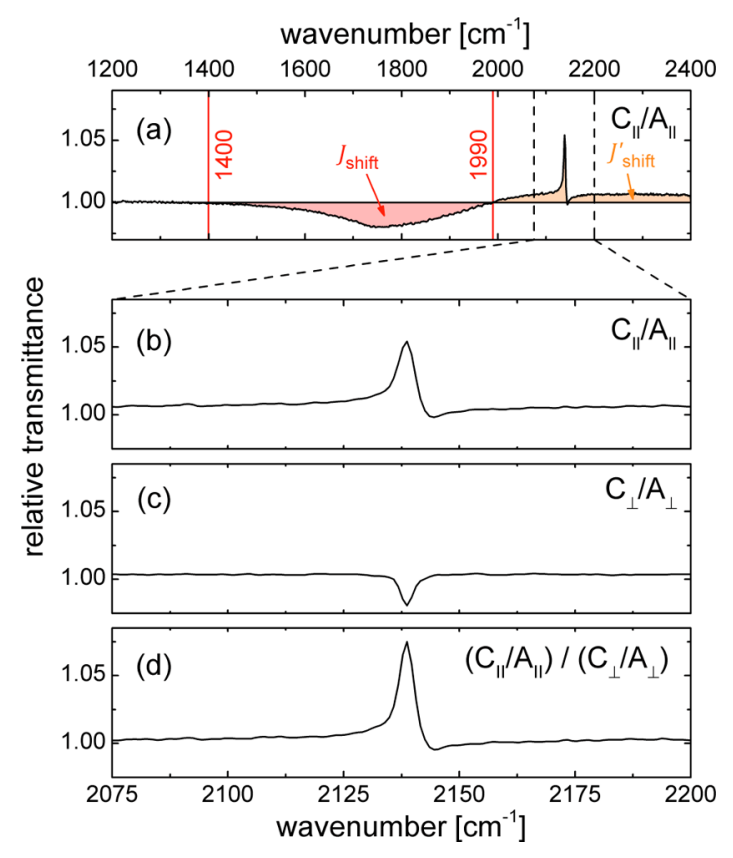

Figure 2. Typical relative transmittance spectra for $d_{\mathrm{Co}}=$ $29.5 \AA$, with the uncovered antennas as reference and the light polarized parallel to the antennas $\left(\mathrm{C}_{\|} / \mathrm{S}_{\|}\right.$divided by $A_{\|} / S_{\|}$from Figure 1) (a). The area $J_{\text {shift }}$ between 1400 and $1990 \mathrm{~cm}^{-1}$ was integrated to gain information about the shift of the plasmon resonance. Zoom-in of the CO vibrational signal (b). The vibrational signal of $\mathrm{CO}$ adsorbed in between the antennas and on the backside of the substrate is not enhanced by the LSPR (c) and is measured with perpendicular polarization $\left(C_{\perp} / S_{\perp}\right.$ divided by $A_{\perp} / S_{\perp}$ from Figure 1). The quotient of the two previous spectra shows solely the antenna-enhanced signal (d).

characteristic asymmetric line shape; the other fraction originates from CO located sufficiently apart from the enhanced near-fields of the antennas. Most of the CO adsorbed on the sample is actually located in the area between the antennas and on the backside of the substrate and contributes considerably to the total signal. We measure this signal with $\perp$ polarization as shown in Figure 1. To be consistent with the previous spectrum, we normalize $C_{\perp}$ to $A_{\perp}$ (Figure $2 c$ ). Since we are interested only in the antenna-enhanced signal, we divide the superposed signal from Figure $2 b$ by the not enhanced signal from Figure $2 \mathrm{c}$ and obtain, in Figure $2 \mathrm{~d}$, solely the near-field-enhanced CO signal. In the following, all spectra referring to the antenna-enhanced vibrational signal were treated this way. They are displayed in Figure 3 a for different CO layer thicknesses.

As mentioned above, the enhanced signal originates from areas very close to the antennas' surfaces, thus from $\mathrm{CO}$ adsorbed on gold. For comparison to a unenhanced system with the same adsorption behavior, we look at $\mathrm{CO}$ adsorbed on a flat gold surface under exactly the same experimental conditions. The spectra were recorded in reflection geometry under an angle of incidence of $72.5^{\circ}$ and normalized to the spectrum of the same gold substrate before exposing to $\mathrm{CO}$ gas (Figure 3b). 
(a)

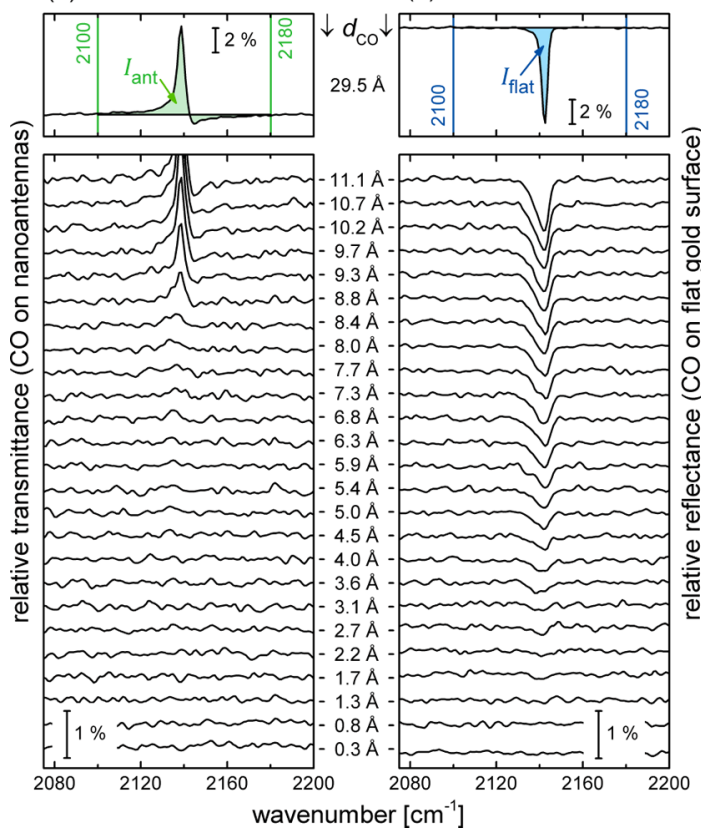

Figure 3. Development of the CO vibrational signal with growing layer thickness on gold antennas (a) and on a flat gold surface (b). Relative transmittance spectra in (a) are corrected for the $\mathrm{CO}$ adsorbed on the $\mathrm{Si}$ substrate, as described in Figure 2, with the spectrum at $d_{\mathrm{CO}}=29.5 \AA$ being the one from Figure 2d. Relative reflectance spectra of CO adsorbed on a flat gold surface are shown as measured (b).

The antenna-enhanced signal (Figure 3a) develops for $d_{\mathrm{CO}}>8 \AA$ and exhibits the Fano-line shape, typical for coupled systems. In contrast to that, the unenhanced signal (Figure 3b) is already visible for much thinner CO layers and shows a slightly asymmetric line shape, which can be explained by a vibrational dipole interaction in disordered systems. ${ }^{37}$ Even though the CO vibrational signal is of similar size for $d_{C O}=29.5 \AA$, it is striking that it develops very differently for the two systems.

Signal Evolution. To point out this effect in more detail, we integrated the absolute peak area from 2100 to $2180 \mathrm{~cm}^{-1}$ of the antenna-enhanced signal $\left(l_{\text {ant }}\right)$ as well as the unenhanced signal of $\mathrm{CO}$ on the flat gold surface $\left(I_{\text {flat }}\right)$. The results are presented in Figure $4 a$, together with $\Delta \omega_{\text {res }}$ as derived earlier.

While the unenhanced $I_{\text {flat }}$ rises linearly with the CO layer thickness, indicating a constant adsorption rate of CO on gold, we observe no antenna-enhanced signal up to about $8 \AA$ and a rapid increase for thicker layers. Clearly, the shift of the LSPR frequency $\Delta \omega_{\text {res }}$ behaves very similarly, indicating that not only the $\mathrm{CO}$ vibration but also the antennas themselves are not sensitive to the first few CO layers. The identical feature of the two curves is strong evidence of the plasmonic nature of the observed effect.

Delayed adsorption of $\mathrm{CO}$ on gold or adsorption of $\mathrm{CO}$ with the dipole vector parallel to the surface, making it invisible for IR radiation, ${ }^{38}$ can be excluded

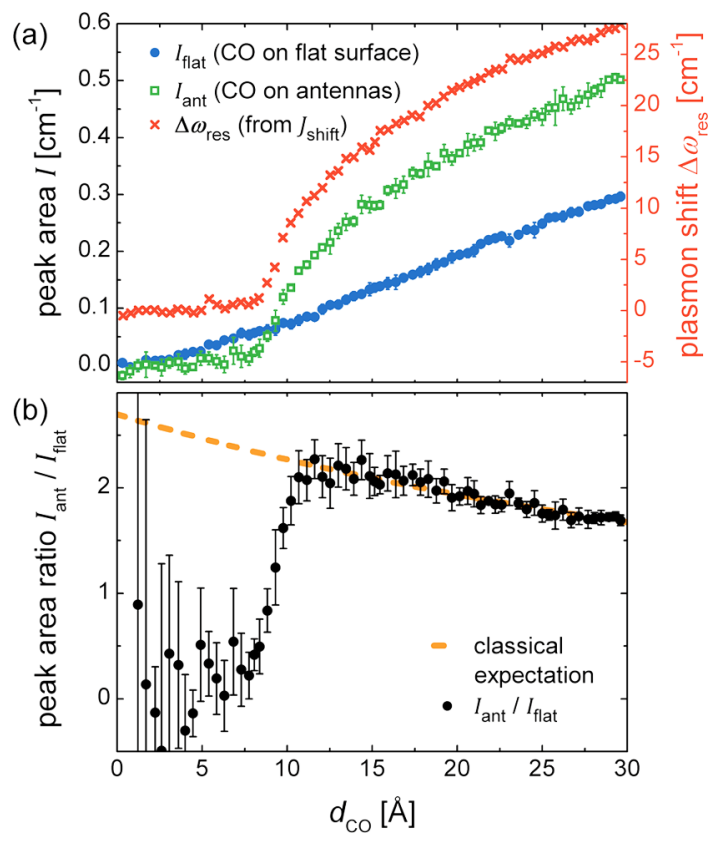

Figure 4. Peak area (integrated from 2100 to $2180 \mathrm{~cm}^{-1}$, see Figure 3) of the vibrational signal of $\mathrm{CO}$ on a flat gold surface ( $I_{\text {flat }}$, blue), absolute peak area of the Fano-type signal of CO on the antennas ( $l_{\text {ant }}$, green), and the shift of the plasmon resonance ( $\Delta \omega_{\text {res, }}$, red) are shown over the CO layer thickness $d_{\mathrm{CO}}(\mathrm{a})$. Please note the different ordinate offsets. The ratio of $I_{\text {ant }}$ to $I_{\text {flat }}$, which is proportional to the signal enhancement induced by the antennas, is displayed in (b) together with a fit to the data above $15 \AA$, which describes the expected behavior according to classical electrodynamics.

due to the linear growth observed for $\mathrm{CO}$ on a flat gold surface (Figure 4a). We assume comparable surface roughness of the antennas and the flat gold surface due to the similar evaporation process and the annealing above $290 \mathrm{~K}^{39}$ of both samples. Surface contamination by remaining resist from the fabrication can also be excluded since the strong vibrational mode of the used resist at $1726 \mathrm{~cm}^{-1}$ would also be enhanced by the antennas and appear in Figure 1.

However, a quantum mechanically explained spillout of the conduction electrons, suppressing the local near-field on the first few angstroms, ${ }^{23}$ could explain both the shift of the LSPR frequency and the trace of the $\mathrm{CO}$ vibrational signal. Knowing these results, we take another look at the ALD results ${ }^{30}$ mentioned above. We notice that, especially for the particles with the highest aspect ratio, the data points below $7 \AA$ are systematically lower. This effect is not mentioned in their discussion, but confirms our findings on a system with significant experimental differences. Additionally, earlier unpublished work on ethylene adsorbed on copper islands also indicates a very similar increase of IR signals with adsorbate layer thickness [M. Sinther, A. Pucci, unpublished data].

To point out the different signal evolutions in more detail, the ratio between the antenna-enhanced signal and the signal on a flat gold surface is displayed in Figure $4 \mathrm{~b}$. The decay of the signal enhancement above 
$15 \AA$ is described within classical electrodynamic approximations, ${ }^{40,41}$ and the result is shown as the dashed line in Figure 4b. A detailed discussion can be found in the Supporting Information.

Considering the amount of molecules actively contributing to the vibrational signal, the intrinsic signal enhancement of the flat gold surface compared to a transparent substrate, and the thickness of a monolayer, we deduce that the antennas enhance the vibrational signal of the $\mathrm{CO}$ molecule up to a factor of 55000 . Technical details are again given in the Supporting Information.

Our findings confirm the quantum mechanically expected influence of the electron spill-out on the signal enhancement. Even though the affected distances we found are larger than the ones theoretically predicted for very small antennas, ${ }^{23}$ we can explain these differences by effects such as the closer spacing of the electronic states in bigger particles, the electron image potential which was neglected in the theoretical work, and particles' roughness, which are all predicted to increase the range of decreased near-field. ${ }^{23}$

\section{CONCLUSION}

With the IR signal size being related to the local electromagnetic field, we presented a system in this work that allows measuring the distance dependence of
LSPR-induced near-fields with Angstrom-scale resolution. To achieve this, we cold-condensed $\mathrm{CO}$ on the surface of gold antennas featuring LSPRs in the infrared and observed the evolution of the vibrational signal with growing layer thickness. We found no signal enhancement within the first $8 \AA$ from the surface and could describe the signal size of layers thicker than $15 \AA$ by a classical model. The classical model predicts the highest field, and thus the highest signal enhancement, at the surface of the antenna, which is in clear contrast to our measurement results, whereas quantum mechanical calculations show a behavior that can explain our results.

Using the same experimental conditions and materials, we have shown that $\mathrm{CO}$ physisorbs with constant adsorption rate on flat gold surfaces, which do not feature plasmonic enhancement. In contrast to the antenna-enhanced case, the thereby measured increase of the $\mathrm{CO}$ vibrational signal is linear, as expected.

Additionally to our experiments, we found other works in the field of plasmonic sensing, which show evidence of a decreased near-field close to the surface of copper island films as well as plasmonic silver triangles. Furthermore, our results could also explain the missing antenna-enhanced vibrational water bands of the omnipresent water layer when working in ambient conditions.

\section{METHODS}

Gold Antennas. The gold antennas were prepared on a silicon substrate with a natural $\mathrm{SiO}_{2}$ layer by standard electron beam lithography (EBL), which is described elsewhere. ${ }^{42}$ The antennas are $100 \mathrm{~nm}$ wide and high and $800 \mathrm{~nm}$ long and feature the dipolar LSPR frequency at about $1950 \mathrm{~cm}^{-1}$, close to the CO stretching vibration. They are arranged in a $4 \times 4 \mathrm{~mm}$ array, containing over 1.7 million antennas, with interantenna distances of 1 and $5 \mu \mathrm{m}$, parallel and perpendicular to the antennas' long axis, respectively. The spacing prevents coupling of the antennas, ${ }^{43}$ and the overall array size is adjusted to the IR geometry (spot size $3.8 \mathrm{~mm}$ in diameter, near parallel beam with $\mathrm{NA}=0.08$ ) inside the vacuum chamber, allowing in situ measurements to exclude misalignment after separate growing and measuring steps.

Flat Gold Surface. The flat gold surface was also prepared on a silicon substrate to guarantee similar heat transfer during cooling. It is $200 \mathrm{~nm}$ thick, has a rms roughness of about $4 \mathrm{~nm}$, and was purchased from Georg Albert Physikal Vapor Disposition.

Experimental Setup. The antenna sample was mounted on a liquid He cooled cryostat inside a UHV chamber. This allows cooling the sample to about $18 \mathrm{~K}$, to enable multilayer physisorption of $\mathrm{CO} .{ }^{44}$ At this temperature CO forms fcc crystals with a lattice plane spacing of $1.6 \AA$ in the (111) direction, ${ }^{45}$ allowing angstrom-scale resolution. The sample holder supports only the edges of the silicon wafer and allows in situ transmission measurements while exposing $\mathrm{CO}$. For in situ reflection measurements the sample holder can be turned to a fixed angle of incidence of $72.5^{\circ}$. A linear polarizer is placed between the FTIR spectrometer (Bruker Tensor 27) and the chamber, allowing measurements with defined polarization. The IR radiation is coupled in and out of the chamber through $\mathrm{KBr}$ windows and detected with a liquid nitrogen cooled MCT detector. All spectra were taken with a spectral resolution of $2 \mathrm{~cm}^{-1}$.
The base pressure inside the UHV chamber was below $10^{-10}$ mbar, and CO gas was fed into the chamber via a leak valve, achieving a partial pressure of $10^{-8}$ mbar. The CO purity of $99.997 \%$ was also checked with mass spectrometry.

Experimental Procedure. Before opening the CO leak valve, the sample temperature was quickly raised to $370 \mathrm{~K}$ via electron bombardment of the sample holder, to desorb unwanted species that were obtained during cooling. Afterward the temperature dropped to $18 \mathrm{~K}$ in less than a minute, and a spectrum of the cooled sample was taken with the respective polarization. The CO leak valve was opened, and IR spectra were taken in situ during CO film growth. All following spectra were divided by one of these reference spectra. This procedure was repeated for polarization parallel and perpendicular to the antennas and for the flat gold surface in p-polarization.

Conflict of Interest: The authors declare no competing financial interest.

Acknowledgment. We would like to thank the Deutsche Forschungsgemeinschaft (DFG Pu 193/9), the Japanese Science Foundation (Strategic International Cooperative Program), and Grant-in-Aid for Scientific Research from the Ministry of Education, Culture, Sports, Science, and Technology (MEXT) for financial support. Part of this work was supported by the World Premier International Research Center (WPI) Initiative on Materials Nanoarchitectonics, MEXT, Japan. We gratefully thank D. Enders and the MANA foundry station for the EBL preparation of the antenna samples. J.B. and F.N. acknowledge funding within the "Nanoantenna" collaborative European project (No. HEALTH-F5-2009-241818). J.B. acknowledges support from the Heidelberg Graduate School of Fundamental Physics.

Supporting Information Available: The method to determine the thickness of the adsorbed CO layer, the calculation of the enhancement factor, and the classical model in Figure 4 (b). This 
material is available free of charge via the Internet at http:// pubs.acs.org.

\section{REFERENCES AND NOTES}

1. Nie, S.; Emory, S. R. Probing Single Molecules and Single Nanoparticles by Surface-Enhanced Raman Scattering. Science 1997, 275, 1102-1106.

2. Theiss, J.; Pavaskar, P.; Echternach, P. M.; Muller, R. E.; Cronin, S. B. Plasmonic Nanoparticle Arrays with Nanometer Separation for High-Performance SERS Substrates. Nano Lett. 2010, 10, 2749-2754.

3. Kneipp, K.; Wang, Y.; Kneipp, H.; Perelman, L. T.; Itzkan, I.; Dasari, R. R.; Feld, M. S. Single Molecule Detection Using Surface-Enhanced Raman Scattering (SERS). Phys. Rev. Lett. 1997, 78, 1667-1670.

4. Fazio, B.; D'Andrea, C.; Bonaccorso, F.; Irrera, A.; Calogero, G.; Vasi, C.; Gucciardi, P. G.; Allegrini, M.; Toma, A.; Chiappe, D.; et al. Re-radiation Enhancement in Polarized SurfaceEnhanced Resonant Raman Scattering of Randomly Oriented Molecules on Self-Organized Gold Nanowires. ACS Nano 2011, 5, 5945-5956.

5. Neubrech, F.; Pucci, A.; Cornelius, T. W.; Karim, S.; GarciaEtxarri, A.; Aizpurua, J. Resonant Plasmonic and Vibrational Coupling in a Tailored Nanoantenna for Infrared Detection. Phys. Rev. Lett. 2008, 101, 157403.

6. Adato, R.; Yanik, A. A.; Amsden, J. J.; Kaplan, D. L.; Omenetto, F. G.; Hong, M. K.; Erramilli, S.; Altug, H. Ultra-Sensitive Vibrational Spectroscopy of Protein Monolayers with Plasmonic Nanoantenna Arrays. Proc. Natl. Acad. Sci. 2009, 106, 19227-19232.

7. Cataldo, S.; Zhao, J.; Neubrech, F.; Frank, B.; Zhang, C.; Braun, P. V.; Giessen, H. Hole-Mask Colloidal Nanolithography for Large-Area Low-Cost Metamaterials and Antenna-Assisted Surface-Enhanced Infrared Absorption Substrates. ACS Nano 2012, 6, 979-985.

8. Kinkhabwala, A.; Yu, Z.; Fan, S.; Avlasevich, Y.; Mullen, K.; E., M. Large Single-Molecule Fluorescence Enhancements Produced by a Bowtie Nanoantenna. Nat. Photonics 2009, 3, 654-657

9. Bardhan, R.; Grady, N. K.; Cole, J. R.; Joshi, A.; Halas, N. J. Fluorescence Enhancement by Au Nanostructures: Nanoshells and Nanorods. ACS Nano 2009, 3, 744-752.

10. Vitol, E. A.; Orynbayeva, Z.; Bouchard, M. J.; Azizkhan-Clifford, J.; Friedman, G.; Gogotsi, Y. In Situ Intracellular Spectroscopy with Surface Enhanced Raman Spectroscopy (SERS)-Enabled Nanopipettes. ACS Nano 2009, 3, 3529-3536.

11. Isola, N. R.; Stokes, D. L.; Vo-Dinh, T. Surface-Enhanced Raman Gene Probe for HIV Detection. Anal. Chem. 1998, 70, 1352-1356.

12. Wang, Y.; Lee, K.; Irudayaraj, J. Silver Nanosphere SERS Probes for Sensitive Identification of Pathogens. J. Phys. Chem. C 2010, 114, 16122-16128.

13. Halvorson, R. A.; Vikesland, P. J. Surface-Enhanced Raman Spectroscopy (SERS) for Environmental Analyses. Environ. Sci. Technol. 2010, 44, 7749-7755.

14. Sylvia, J. M.; Janni, J. A.; Klein, J. D.; Spencer, K. M. SurfaceEnhanced Raman Detection of 2,4-Dinitrotoluene Impurity Vapor as a Marker to Locate Landmines. Anal. Chem. 2000, 72, 5834-5840.

15. Schumacher, T.; Kratzer, K.; Molnar, D.; Hentschel, M.; Giessen, H.; Lippitz, M. Nanoantenna-Enhanced Ultrafast Nonlinear Spectroscopy of a Single Gold Nanoparticle. Nat. Commun. 2011, 2, 333.

16. Fernández-Domínguez, A. I.; Wiener, A.; García-Vidal, F. J.; Maier, S. A.; Pendry, J. B. Transformation-Optics Description of Nonlocal Effects in Plasmonic Nanostructures. Phys. Rev. Lett. 2012, 108, 106802.

17. Esteban, R.; Borisov, A. G.; Nordlander, P.; Aizpurua, J. Bridging Quantum and Classical Plasmonics with a QuantumCorrected Model. Nat. Commun. 2012, 3, 825.

18. Marinica, D.; Kazansky, A.; Nordlander, P.; Aizpurua, J.; Borisov, A. G. Quantum Plasmonics: Nonlinear Effects in the Field Enhancement of a Plasmonic Nanoparticle Dimer. Nano Lett. 2012, 12, 1333-1339.
19. Schnell, M.; Garcia-Etxarri, A.; Alkorta, J.; Aizpurua, J.; Hillenbrand, R. Phase-Resolved Mapping of the Near-Field Vector and Polarization State in Nanoscale Antenna Gaps. Nano Lett. 2010, 10, 3524-3528.

20. Hauer, B.; Engelhardt, A. P.; Taubner, T. Quasi-Analytical Model for Scattering Infrared Near-Field Microscopy on Layered Systems. Opt. Express 2012, 20, 13173-13188.

21. Deeb, C.; Bachelot, R.; Plain, J.; Baudrion, A.-L.; Jradi, S.; Bouhelier, A.; Soppera, O.; Jain, P. K.; Huang, L.; Ecoffet, C.; et al. Quantitative Analysis of Localized Surface Plasmons Based on Molecular Probing. ACS Nano 2010, 4, 45794586.

22. Zuloaga, J.; Prodan, E.; Nordlander, P. Quantum Description of the Plasmon Resonances of a Nanoparticle Dimer. Nano Lett. 2009, 9, 887-891.

23. Zuloaga, J.; Prodan, E.; Nordlander, P. Quantum Plasmonics: Optical Properties and Tunability of Metallic Nanorods. ACS Nano 2010, 4, 5269-5276.

24. Feibelman, P. J. Surface Electromagnetic Fields. Prog. Surf. Sci. 1982, 12, 287-407.

25. Plummer, W.; Tsuei, K.-D.; Kim, B.-O. The Impact of the Concept of a Surface Plasmon. Nucl. Instrum. Methods Phys. Res., Sect. B 1995, 96, 448-459.

26. Nagao, T.; Han, G.; Hoang, C.; Wi, J.-S.; Pucci, A.; Weber, D.; Neubrech, F.; Silkin, V. M.; Enders, D.; Saito, O.; et al. Plasmons in Nanoscale and Atomic-Scale Systems. Sci. Technol. Adv. Mater. 2010, 11, 054506.

27. Ament, I.; Prasad, J.; Henkel, A.; Schmachtel, S.; Sönnichsen, C. Single Unlabeled Protein Detection on Individual Plasmonic Nanoparticles. Nano Lett. 2012, 12, 1092-1095.

28. Haes, A. J.; Zou, S.; Schatz, G. C.; Van Duyne, R. P. Nanoscale Optical Biosensor: Short Range Distance Dependence of the Localized Surface Plasmon Resonance of Noble Metal Nanoparticles. J. Phys. Chem. B 2004, 108, 6961-6968.

29. Malinsky, M. D.; Kelly, K. L.; Schatz, G. C.; Van Duyne, R. P. Chain Length Dependence and Sensing Capabilities of the Localized Surface Plasmon Resonance of Silver Nanoparticles Chemically Modified with Alkanethiol Self-Assembled Monolayers. J. Am. Chem. Soc. 2001, 123, 1471-1482.

30. Whitney, A. V.; Elam, J. W.; Zou, S.; Zinovev, A. V.; Stair, P. C.; Schatz, G. C.; Van Duyne, R. P. Localized Surface Plasmon Resonance Nanosensor: A High-Resolution DistanceDependence Study Using Atomic Layer Deposition. J. Phys. Chem. B 2005, 109, 20522-20528.

31. Anger, P.; Bharadwaj, P.; Novotny, L. Enhancement and Quenching of Single-Molecule Fluorescence. Phys. Rev. Lett. 2006, 96, 113002.

32. Kennedy, B. J.; Spaeth, S.; Dickey, M.; Carron, K. T. Determination of the Distance Dependence and Experimental Effects for Modified SERS Substrates Based on Self-Assembled Monolayers Formed Using Alkanethiols. J. Phys. Chem. B 1999, 103, 3640-3646.

33. Otto, A. The 'Chemical' (Electronic) Contribution to SurfaceEnhanced Raman Scattering. J. Raman Spectrosc. 2005, 36, 497-509.

34. Dieringer, J. A.; McFarland, A. D.; Shah, N. C.; Stuart, D. A.; Whitney, A. V.; Yonzon, C. R.; Young, M. A.; Zhang, X.; Van Duyne, R. P. Introductory Lecture Surface Enhanced Raman Spectroscopy: New Materials, Concepts, Characterization Tools, and Applications. Faraday Discuss. 2006, $132,9-26$.

35. Johnson, E.; Aroca, R. Surface-Enhanced Infrared Spectroscopy of Monolayers. J. Phys. Chem. 1995, 99, 9325-9330.

36. Giannini, V.; Francescato, Y.; Amrania, H.; Phillips, C. C.; Maier, S. A. Fano Resonances in Nanoscale Plasmonic Systems: A Parameter-Free Modeling Approach. Nano Lett. 2011, 11, 2835-2840.

37. Persson, B. N. J.; Ryberg, R. Vibrational Interaction Between Molecules Adsorbed on a Metal Surface: The DipoleDipole Interaction. Phys. Rev. B 1981, 24, 6954-6970.

38. Dumas, P.; Tobin, R.; Richards, P. Study of Adsorption States and Interactions of CO on Evaporated Noble Metal Surfaces by Infrared Absorption Spectroscopy: I. Silver. Surf. Sci. 1986, 171, 555-578. 
39. Dumas, P.; Tobin, R.; Richards, P. Interaction of CO Molecules with Evaporated Silver, Gold, and Copper Films: An Infrared Spectroscopic Investigation Using a Thermal Detection Technique. J. Electron Spectrosc. Relat. Phenom. 1986, 39, 183-189.

40. Alonso-Gonzalez, P.; Albella, P.; Schnell, M.; Chen, J.; Huth, F.; Garcia-Etxarri, A.; Casanova, F.; Golmar, F.; Arzubiaga, L.; Hueso, L.; et al. Resolving the Electromagnetic Mechanism of Surface-Enhanced Light Scattering at Single Hot Spots. Nat. Commun. 2012, 3, 684.

41. Cvitkovic, A.; Ocelic, N.; Hillenbrand, R. Analytical Model for Quantitative Prediction of Material Contrasts in ScatteringType Near-Field Optical Microscopy. Opt. Express 2007, 15, 8550-8565.

42. Neubrech, F.; Weber, D.; Enders, D.; Nagao, T.; Pucci, A. Antenna Sensing of Surface Phonon Polaritons. J. Phys. Chem. C 2010, 114, 7299-7301.

43. Weber, D.; Albella, P.; Alonso-González, P.; Neubrech, F.; Gui, H.; Nagao, T.; Hillenbrand, R.; Aizpurua, J.; Pucci, A. Longitudinal and Transverse Coupling in Infrared Gold Nanoantenna Arrays: Long Range Versus Short Range Interaction Regimes. Opt. Express 2011, 19, 15047-15061.

44. Palumbo, M. E.; Baratta, G. A.; Collings, M. P.; McCoustra, M. R. S. The Profile of the $2140 \mathrm{~cm}^{-1}$ Solid CO Band on Different Substrates. Phys. Chem. Chem. Phys. 2006, 8, 279-284.

45. Vegard, I. Struktur und Leuchtfähigkeit von Festem Kohlenoxyd. Z. Phys. A Hadrons Nucl. 1930, 61, 185-190. 